\title{
Phlébotomes du Sénégal (Diptera-Psychodidae) : PEUPLEMENT ET DYNAMIQUE DES POPULATIONS DE LA RÉGION DE MONT-ROLLAND
}

\author{
BA Y.*, TROUILLET J.**, THONNON J.** \& FONTENILLE D.*
}

Summary : POPULATION DYNAMICS OF SANDFLIES (DIPTERAPSYCHODIDAE) FROM SENEGAL (MONT-ROLLAND DisTRICT)

Phlebotomine sandflies were captured on a monthly basis from May 1995 to April 1996 in the Mont-Rolland district in Western Senegal. The objectives were to study the population dymanics of sandflies and to make an inventory of the viruses they transmit. Among 10,315 specimens captured, belonging to 14 species, Sergentomyia dubia $(35.9 \%)$, S. schwetzi $(27.7 \%)$ and S. buxtoni $(24.5 \%)$ were the most abundant. Species from the genus Sergentomyia accounted for $99.6 \%$ versus $0.4 \%$ for the genus Phlebotomus. The sandflies population was observed to peak in February. The most populated resting sites of the captured insects were in decreasing order tree-holes, termite-hills and burrows. S. dubia was the most abundant species captured in tree-holes. It was $S$. buxtoni in termite-hills, while $S$. schwetzi was found to dwell most often in burrows. No virus was isolated from 2,114 specimens tested.

KEY WORDS : sandflies, Phlebotomus, Sergentomyia, ecology, arboviruses, Senegal.

\section{INTRODUCTION}

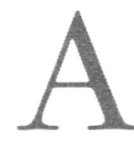

bonnenc \& Larivière (1959), dans leur " Répartition des Phlébotomes de l'Ouest africain ", signalaient la présence au Sénégal de onze espèces de Phlébotomes. De nombreuses autres publications (Abonnenc, 1972; Raymond \& Cornet, 1976; Dedet et al., 1980a; Dedet et al., 1980b; Desjeux \& Waroquy, 1981b; Pastre, 1975; Pastre, 1982; Niang, 1992; Niang \& Trouillet, 1993; Niang \& Trouillet, 1995; Trouillet \& Faye, 1993; Trouillet et al., 1993; Ba, 1994) ont, par la suite, allongé cette liste qui est actuellement

\footnotetext{
* Laboratoire ORSTOM de Zoologie Médicale à l'Institut Pasteur de Dakar, BP 1386, Dakar, Sénégal.

** Université Cheikh Anta Diop, Département de Biologie Animale, Dakar, Sénégal.

*** Institut Pasteur, BP 220, Dakar, Sénégal.

Correspondance : Didier Fontenille.

Tél. : 2218234874 . Fax : 2218399210

E-mail : fontenil@dakar.orstom.sn.
}

Résumé :

Quatorze espèces de Phlébotomes ont été capturées dans la communauté rurale de Mont-Rolland. Les espèces les plus abondantes sont respectivement: Sergentomyia dubia $(35,9 \%)$, S. schwetzi $(27,7 \%)$ et S. buxtoni $(24,5 \%)$. Le genre Sergentomyia constitue $99,6 \%$ des récoltes contre seulement $0,4 \%$ pour le genre Phlebotomus. L'ensemble du peuplement a présenté un maximum d'abondance en février. Les habitats préférentiels des Phlébotomes sont, dans l'ordre d'abondance d'individus capturés les trous d'arbres, les termitières et les terriers. S. dubia est l'espèce dominante dans les trous d'arbres. Elle est remplacée par S. buxtoni dans les termitières et par S. schwetzi dans les terriers.

MOTS CLÉS : phlébotomes, Phlebotomus, Sergentomyia, écologie, arbovirus, Sénégal.

de 29 espèces. Si ces travaux s'inscrivaient en général dans le cadre d'enquête sur les leishmanioses, l'étude de la circulation des arbovirus chez les Phlébotomes du Sénégal est récente (Fontenille et al., 1994; Trouillet et al., 1995). Des arbovirus potentiellement pathogènes pour l'homme, comme Saboya et Chandipura, ont été isolés des Phlébotomes. Notre recherche s'inscrit dans ce double esprit de contribution à l'étude des Phlébotomes du Sénégal et de leur rôle dans la transmission des arbovirus.

\section{MATÉRIELS ET MÉTHODES}

\section{RÉGION ÉTUDIÉE}

L

a communauté rurale de Mont-Rolland est située à l'ouest du Sénégal dans la région de Thiès, à environ $10 \mathrm{~km}$ au nord de cette ville. Nos recherches se sont concentrées sur deux de ses villages : Palo Youga et Palo Dial distants d'environ $1,5 \mathrm{~km}$. Cette zone est située entre les latitudes $14^{\circ} 55^{\prime}$ $14^{\circ} 56^{\prime}$ Nord et les longitudes $16^{\circ} 50^{\prime}-16^{\circ} 55^{\prime}$ Ouest. 
Le climat est de type tropical sahélo-soudanien. La saison des pluies s'étend en général de la fin du mois de juin au début du mois d'octobre. La zone est comprise entre les isohyètes 500 et $650 \mathrm{~mm}$. L'année 1995 avec une hauteur pluviométrique annuelle de $515 \mathrm{~mm}$ est normale tandis que l'année 1996 avec une pluviométrie annuelle de $446 \mathrm{~mm}$ est déficitaire.

La moyenne thermique annuelle est de $26,7^{\circ} \mathrm{C}$ avec un maximum de $29,3{ }^{\circ} \mathrm{C}$ en juillet et un minimum de $24,4^{\circ} \mathrm{C}$ en janvier. L'hygrométrie subit des variations saisonnières et journalières, respectivement maximale en hivernage et pendant la deuxième moitié de la nuit, avec une moyenne de $90 \%$. Les minima s'observent en fin de saison sèche pendant la journée avec une moyenne de $25 \%$.

La végétation est de type savane arbustive à essences épineuses.

Les Mammifères sauvages sont représentés par le genre Ichneumia (Ichneumia albicauda ou Mangouste à queue blanche), les Lagomorphes (Lepus sp.), les Sciuromorphes (Xerus erythropus), les Myomorphes (Taterillus sp., Mastomys erythroleucus).

\section{Techniques de PIÉGeAge} ET BIOTOPES INVENTORIÉS

Deux types de piégeage ont été utilisés :

- des papiers huilés ("PH "). Ce sont des feuilles de papier blanc de $20 \mathrm{~cm}$ de côté enduites d'huile de ricin. Ils sont placés à l'entrée des gîtes de Phlébotomes (terriers de Mangouste à queue blanche et de petits rongeurs non déterminés, termitières et trous d'arbres souvent colonisés par des Geckos, des Serpents, et des Lézards) le soir vers $18 \mathrm{~h}$ au coucher du soleil, et relevés le lendemain matin vers 7 h. Ils permettent d'étudier les fluctuations saisonnières et de comparer les densités de peuplement des différents biotopes;

- des pièges lumineux de type CDC avec carboglace pour obtenir les récoltes nécessaires à la recherche des arbovirus et pour mieux connaître la représentativité des espèces vivant dans la litière ou la végétation herbacée. Ils ont été placés à environ 2 mètres de gîtes offrant un bon rendement avec les papiers huilés.

Les récoltes de Phlébotomes ont été effectuées à proximité des villages de Palo Youga et de Palo Dial. Le piégeage adhésif " $\mathrm{PH}$ " a été réalisé chaque mois au cours de deux nuits successives de mai 1995 à avril 1996 à Palo Youga. Le piégeage lumineux CDC a été réalisé chaque mois au cours de deux nuits successives de mai 1995 à octobre 1996 à Palo Dial et de mai 1995 à novembre 1996 à Palo Youga. Trois pièges CDC sont placés chaque nuit dans chacun de ces deux villages.

\section{MonTAge}

Les Phlébotomes identifiés ont fait l'objet d'un montage permanent à l'Euparal, après éclaircissement dans une solution de potasse à $20 \%$, rinçage à l'eau distillée, mordançage dans le liquide de Marc André, déshydratation par passage dans l'alcool absolu pendant $1 \mathrm{~h}$. Certains spécimens ont été montés directement dans du Diméthyl hydantoïne formaldéhyde (DMHF) (Bameul, 1990).

La détermination des spécimens a été faite à l'aide des travaux de Abonnenc (1972) et de Davidson (1990).

\section{ANALYSE DES RÉSULTATS}

Quatre caractéristiques de la faune phlébotomienne ont été déterminées :

- l'abondance : nombre relatif des individus de chaque espèce par rapport à l'ensemble des Phlébotomes récoltés;

- la richesse spécifique : nombre d'espèces que compte le peuplement;

- la densité : nombre d'individus par unité de surface de papier huilé. Elle est exprimée en nombre de Phlébotomes par $\mathrm{m}^{2}$ (phl./ $\left.\mathrm{m}^{2}\right)$;

- la fréquence : rapport, exprimé sous la forme du pourcentage $\mathrm{F}=(\mathrm{p} \times 100) / \mathrm{P}$, dans lequel $\mathrm{p}$ est le nombre de mois où une espèce donnée a été récoltée et $\mathrm{P}$, le nombre de mois d'étude. En fonction de la valeur de $\mathrm{F}$, on distingue :

- des espèces très communes ( $\mathrm{F}$ est supérieure à $50 \%)$;

- des espèces communes ( $\mathrm{F}$ est comprise entre 25 et $50 \%)$;

- des espèces rares (F est comprise entre 10 et $25 \%$ );

- des espèces très rares ( $\mathrm{F}$ est inférieure à $10 \%$ ).

\section{RECHERCHE D'ARBOVIRUS}

Des lots de Phlébotomes non déterminés constitués à partir des captures aux pièges lumineux ont été broyés dans du milieu de Hanks albumine, centrifugés et inoculés à des souriceaux nouveau-nés et à des lignées de cellules de Moustique Aedes pseudoscutellaris (AP 61) pour recherche d'arbovirus.

\section{RÉSULTATS}

\section{RÉSULTATS GLOBAUX}

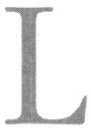
es deux techniques de capture ont permis la récolte de 10315 Phlébotomes appartenant à 14 espèces : Phlebotomus (Phlebotomus) duboscqi Neveu-Lemaire, 1906; Pblebotomus (Anaphlebotomus) rodhaini Parrot, 1930; Sergentomyia (Grassomyia) ghesquierei (Parrot, 1929); Sergentomyia 
Ensemble des biotopes

\begin{tabular}{|c|c|c|c|c|c|c|c|c|c|}
\hline \multirow[b]{2}{*}{ Genres } & \multirow[b]{2}{*}{ Sous-genres } & \multirow[b]{2}{*}{ Espèces } & \multirow[b]{2}{*}{ Nb. Phl. } & \multicolumn{3}{|c|}{ Densité $\left(\mathrm{Phl} . / \mathrm{m}^{2}\right)$} & \multicolumn{3}{|c|}{ Abondance en \% } \\
\hline & & & & Espèces & $\begin{array}{l}\text { Sous- } \\
\text { genres }\end{array}$ & Genres & Espèces & $\begin{array}{l}\text { Sous- } \\
\text { genres }\end{array}$ & Genres \\
\hline \multirow[t]{2}{*}{ Pblebotomus } & Anapblebotomus & rodhaini & 24 & 0,63 & 0,63 & \multirow[b]{2}{*}{0,99} & 0,24 & 0,24 & \multirow[b]{2}{*}{0,38} \\
\hline & Phlebotomus & duboscqi & 14 & 0,36 & 0,36 & & 0,14 & 0,14 & \\
\hline \multirow[t]{9}{*}{ Sergentomyia } & Grassomyia & ghesquierei & 1 & 0,03 & & \multirow{9}{*}{256,87} & 0,01 & & \multirow{9}{*}{99,62} \\
\hline & & squamipleuris & 1 & 0,03 & 0,05 & & 0,01 & 0,02 & \\
\hline & Parrotomyia & magna & 292 & 7,60 & 7,60 & & 2,95 & 2,95 & \\
\hline & Sergentomyia & antennata & 84 & 2,19 & & & 0,85 & & \\
\hline & & buxtoni & 2427 & 63,20 & & & 24,51 & & \\
\hline & & dubia & 3555 & 92,58 & & & 35,90 & & \\
\hline & & schwetzi & 2746 & 71,51 & 229,48 & & 27,73 & 88,99 & \\
\hline & Sintonius & adleri & 600 & 15,63 & & & 6,06 & & \\
\hline & & clydei & 158 & 4,11 & 19,74 & & 1,60 & 7,66 & \\
\hline \multicolumn{3}{|l|}{ Total } & 9902 & 257,86 & 257,86 & & 100,00 & & \\
\hline
\end{tabular}

Nb. Phl. $=$ Nombre de phlébotomes. Surface $=480$ pièges ou $38,4 \mathrm{~m}^{2}$.

Tableau I. - Effectif, densité et abondance dans l'ensemble des biotopes d'après les captures sur papiers huilés " PH ".

(Grassomyia) inermis (Theodor, 1938); Sergentomyia (Grassomyia) squamipleuris (Newstead, 1912); Sergentomyia (Sintonius) adleri (Theodor, 1933); Sergentomyia (Sintonius) affinis vorax (Parrot, 1948); Sergentomyia (Sintonius) clydei (Sinton, 1928); Sergentomyia (Sergentomyia) antennata (Newstead, 1912); Sergentomyia (Sergentomyia) buxtoni (Theodor, 1933); Sergentomyia (Sergentomyia) distincta (Theodor, 1933); Sergentomyia (Sergentomyia) dubia (Parrot, Mornet \& Cadenat, 1945); Sergentomyia (Sergentomyia) schwetzi (Adler, Theodor \& Parrot, 1929), var. typicus \& atypicus; Sergentomyia (Parrotomyia) magna (Sinton, 1932).

\section{Faune phlébotomienne de Palo Youga CAPTURÉE SUR PIÈGES ADHÉSIFS ("PH ")}

Cette méthode a permis la capture de 9902 Phlébotomes appartenant à onze espèces. Il existe des variations de densité globale entre les trous d'arbres d'une part, les terriers et les termitières d'autre part.

Certaines espèces sont plus inféodées à un biotope donné. La richesse spécifique, l'abondance, la densité et la fréquence des Phlébotomes capturés sur " $\mathrm{PH}$ " sont représentées tableaux I, II et III.

\section{FLUCTUATIONS SAISONNIÈRES}

La courbe d'abondance de l'ensemble du peuplement croît dès les premières pluies, atteint un premier pic en décembre, chute en janvier, passe par un maximum en février puis régresse. Les fluctuations du peuplement et des espèces $S$. dubia, $S$. buxtoni et $S$. schwetzi sont indiquées dans la figure 1.

\section{FAUNE PHLÉBOTOMIENNE CAPTURÉE \\ AVEC LES PIÈGES LUMINEUX CDC}

La richesse spécifique, l'abondance et la fréquence des Phlébotomes capturés au piège lumineux sont présentées tableau IV.

\section{RECHERCHE D'ARBOVIRUS}

Les récoltes effectuées aux pièges lumineux CDC ont permis de constituer 21 lots, soit 2114 phlébotomes

\begin{tabular}{lccc}
\hline & \multicolumn{3}{c}{ Fréquence en \% } \\
\cline { 2 - 4 } \multicolumn{1}{c}{ Espèces } & Termitières & Terriers & Trous d'arbres \\
\hline P. rodhaini & 16,7 & 58,3 & 16,7 \\
P. duboscqi & 16,7 & 33,3 & 25,0 \\
S. ghesquierei & 0,0 & 0,0 & 8,3 \\
S. squamipleuris & 0,0 & 0,0 & 8,3 \\
S. magna & 66,7 & 100,0 & 91,7 \\
S. antennata & 33,3 & 66,7 & 33,3 \\
S. buxtoni & 100,0 & 91,7 & 100,0 \\
S. dubia & 83,3 & 91,7 & 100,0 \\
S. schwetzi & 100,0 & 100,0 & 100,0 \\
S. adleri & 100,0 & 100,0 & 91,7 \\
S. clydei & 66,7 & 91,7 & 58,3 \\
\hline
\end{tabular}

Tableau III. - Fréquence des espèces récoltées sur papiers huilés dans les trois biotopes prospectés à Palo Youga 


\begin{tabular}{|c|c|c|c|c|c|c|c|c|c|c|c|c|c|c|c|c|c|}
\hline \multirow[b]{3}{*}{ Genres } & \multicolumn{2}{|c|}{$\begin{array}{c}\text { Biotopes } \\
\text { Surface }\end{array}$} & \multicolumn{5}{|c|}{$\begin{array}{l}\text { Termitières } \\
S=9,6 \mathrm{~m}^{2}\end{array}$} & \multicolumn{5}{|c|}{$\begin{array}{c}\text { Terriers } \\
S=19,2 \mathbf{m}^{2}\end{array}$} & \multicolumn{5}{|c|}{$\begin{array}{l}\text { Trous d'arbres } \\
\qquad s=9,6 \mathrm{~m}^{2}\end{array}$} \\
\hline & \multirow[b]{2}{*}{ Sous-genres } & \multirow[b]{2}{*}{ Espèces } & \multirow[b]{2}{*}{ Nb. Phl. } & \multicolumn{2}{|c|}{ Densité $\left(\mathrm{Phl} / . \mathrm{m}^{2}\right)$} & \multicolumn{2}{|c|}{ Abondance en $\%$} & \multirow[b]{2}{*}{ Nb. Phl. } & \multicolumn{2}{|c|}{ Densité (Phl./m²) } & \multicolumn{2}{|c|}{ Abondance en $\%$} & \multirow[b]{2}{*}{ Nb. Phl. } & \multicolumn{2}{|c|}{ Densité (Phl./m²) } & \multicolumn{2}{|c|}{ Abondance en $\%$} \\
\hline & & & & Espèces & $\begin{array}{l}\text { Sous- } \\
\text { genres }\end{array}$ & Espèces & $\begin{array}{l}\text { Sous- } \\
\text { genres }\end{array}$ & & Espèces & $\begin{array}{l}\text { Sous- } \\
\text { genres }\end{array}$ & Espèces & $\begin{array}{l}\text { Sous- } \\
\text { genres }\end{array}$ & & Espèces & $\begin{array}{l}\text { Sous- } \\
\text { genres }\end{array}$ & Espèces & $\begin{array}{l}\text { Sous- } \\
\text { genres }\end{array}$ \\
\hline \multirow{2}{*}{ Pblebotomus } & Anaphlebotomus & rodhaini & 3 & 0,31 & 0,31 & 0,14 & 0,14 & 18 & 0,94 & 0,94 & 0,42 & 0,42 & 3 & 0,31 & 0,31 & 0,09 & 0,09 \\
\hline & Pblebotomus & duboscqi & 2 & 0,21 & 0,21 & 0,09 & 0,09 & 8 & 0,42 & 0,42 & 0,19 & 0,19 & 4 & 0,42 & 0,42 & 0,12 & 0,12 \\
\hline \multirow[t]{4}{*}{ Sergentomyia } & Grassomyia & $\begin{array}{l}\text { ghesquierei } \\
\text { squamipleuris }\end{array}$ & $\begin{array}{l}0 \\
0\end{array}$ & $\begin{array}{l}0,00 \\
0,00\end{array}$ & 0,00 & $\begin{array}{l}0,00 \\
0,00\end{array}$ & 0,00 & 0 & $\begin{array}{l}0,00 \\
0,00\end{array}$ & 0,00 & $\begin{array}{l}0,00 \\
0,00\end{array}$ & $\begin{array}{l}0,00 \\
0,00\end{array}$ & $\begin{array}{l}1 \\
1\end{array}$ & $\begin{array}{l}0,10 \\
0,10\end{array}$ & 0,20 & $\begin{array}{l}0,03 \\
0,03\end{array}$ & 0,06 \\
\hline & Parrotomyia & magna & 31 & 3,23 & 3,23 & 1,44 & 1,44 & 115 & 5,99 & 5,99 & 2,68 & 2,68 & 146 & 15,21 & 15,21 & 4,21 & 4,21 \\
\hline & Sergentomyia & $\begin{array}{l}\text { antennata } \\
\text { buxtoni } \\
\text { dubia } \\
\text { schwetzzi }\end{array}$ & $\begin{array}{r}11 \\
1474 \\
166 \\
331\end{array}$ & $\begin{array}{r}1,15 \\
153,54 \\
17,29 \\
34,48\end{array}$ & 206,46 & $\begin{array}{r}0,51 \\
68,43 \\
7,71 \\
15,37\end{array}$ & 92,02 & $\begin{array}{r}65 \\
698 \\
910 \\
1937\end{array}$ & $\begin{array}{r}3,38 \\
36,35 \\
47,39 \\
100,88\end{array}$ & 188,00 & $\begin{array}{r}1,51 \\
16,27 \\
21,21 \\
45,14\end{array}$ & 84,13 & $\begin{array}{r}8 \\
255 \\
2479 \\
478\end{array}$ & $\begin{array}{r}0,83 \\
26,56 \\
258,23 \\
49,79\end{array}$ & 335,41 & $\begin{array}{r}0,23 \\
7,38 \\
71,71 \\
13,83\end{array}$ & 93,15 \\
\hline & Sintonius & $\begin{array}{l}\text { adleri } \\
\text { clydei }\end{array}$ & $\begin{array}{r}114 \\
22\end{array}$ & $\begin{array}{r}11,88 \\
2,29\end{array}$ & 14,17 & $\begin{array}{l}5,29 \\
1,02\end{array}$ & 6,31 & $\begin{array}{l}436 \\
104\end{array}$ & $\begin{array}{r}22,71 \\
5,42\end{array}$ & 28,13 & $\begin{array}{r}10,16 \\
2,42\end{array}$ & 12,58 & $\begin{array}{l}50 \\
32\end{array}$ & $\begin{array}{l}5,21 \\
3,33\end{array}$ & 8,54 & $\begin{array}{c}1,44 \\
0,93\end{array}$ & 2,37 \\
\hline Total & & & 2154 & 224.38 & & 100,00 & & 4291 & 223,48 & & 100,00 & & 3457 & 360,09 & & 100,00 & \\
\hline
\end{tabular}

Nb. Phl. = Nombre de phlébotomes. Termitières : 120 pièges ou $9,6 \mathrm{~m}^{2}$. Terriers : 240 pièges ou 19,2 $\mathrm{m}^{2}$. Trous d'arbres : 120 pièges ou $9,6 \mathrm{~m}^{2}$

Tableau II. - Effectif, densité et abondance relative des espèces en fonction des biotopes d'après les captures sur papiers huilés " PH " 


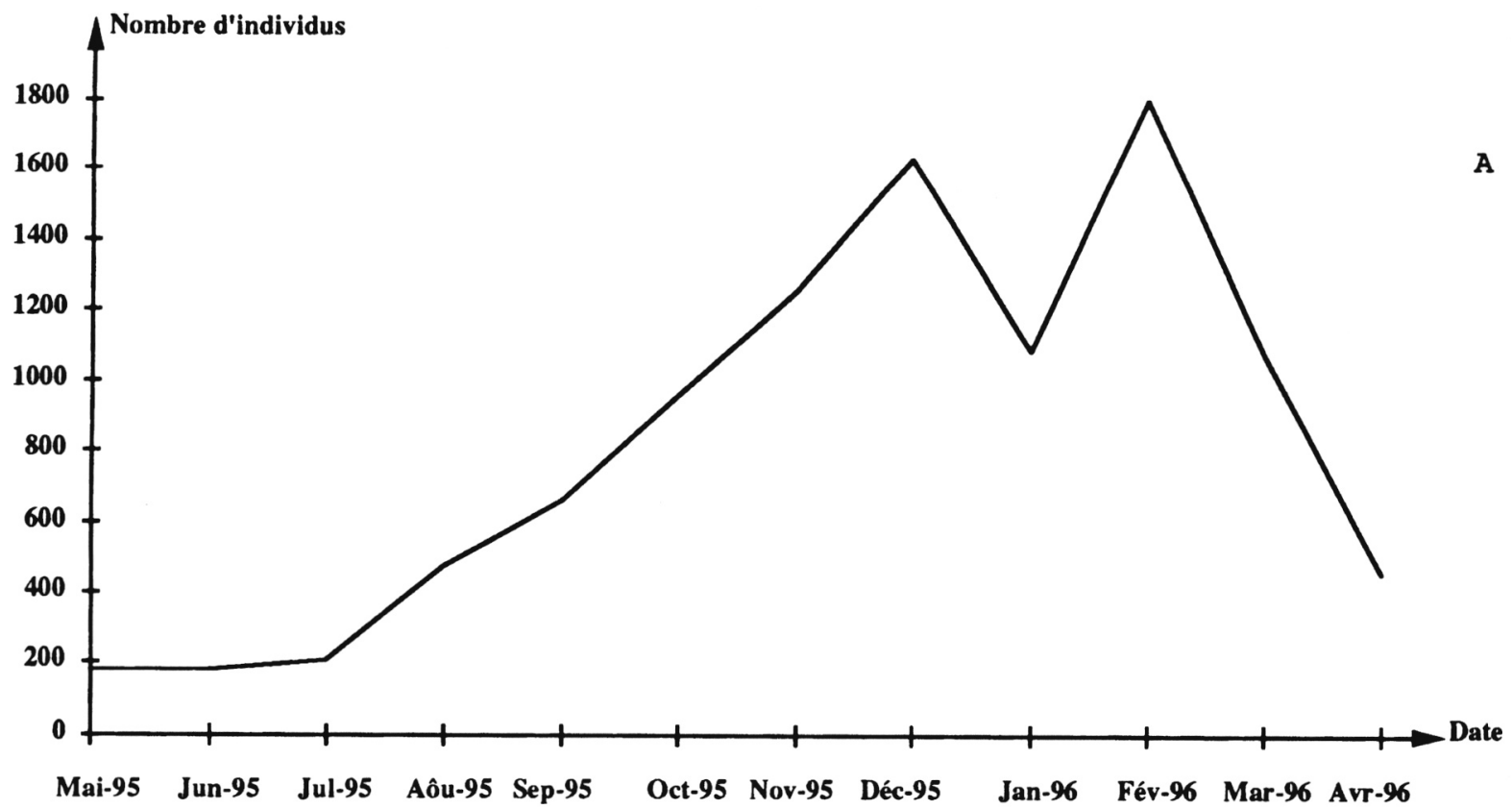

B



Mai-95 Jun-95 Jul-95- Aôu-95 Sep-95 Oct--95 Nov-95 Déc-95 Jan-96 Fév-96 Mar-96 Avr-96



Fig. 1. - A. Fluctuations saisonnières du peuplement de Palo Youga d'après les captures des " $\mathrm{PH}$ ". B. Fluctuations saisonnières des principales espèces d'après les captures des " PH ». C. Diagramme ombrothermique de Mont-Rolland. 


\begin{tabular}{|c|c|c|c|c|c|c|c|}
\hline \multirow[b]{2}{*}{ Espèces } & \multicolumn{2}{|c|}{ Palo Dial } & \multicolumn{2}{|c|}{ Palo Youga } & \multicolumn{2}{|r|}{ Total } & \multirow[b]{2}{*}{ Fréquence en \% } \\
\hline & Nb. Phl. & Abondance en $\%$ & Nb. Phl. & Abondance en $\%$ & Nb. Phl. & Abondance en \% & \\
\hline P. rodhaini & 0 & 0,00 & 2 & 0,68 & 2 & 0,48 & 28,5 \\
\hline S. ghesquierei & 2 & 1,65 & 1 & 0,34 & 3 & 0,73 & 14,2 \\
\hline S. inermis & 3 & 2,48 & 23 & 7,88 & 26 & 6,30 & 28,5 \\
\hline S. squamipleuris & 5 & 4,13 & 24 & 8,22 & 29 & 7,02 & 57,1 \\
\hline S. magna & 3 & 2,48 & 18 & 6,16 & 21 & 5,08 & 42,8 \\
\hline S. antennata & 2 & 1,65 & 2 & 0,68 & 4 & 0,97 & 14,2 \\
\hline S. buxtoni & 12 & 9,92 & 14 & 4,79 & 26 & 6,30 & 85,7 \\
\hline S. distincta & 0 & 0,00 & 1 & 0,34 & 1 & 0,24 & 14,2 \\
\hline S. dubia & 46 & 38,02 & 72 & 24,66 & 118 & 28,57 & 100,0 \\
\hline S. schwetzi & 44 & 36,36 & 118 & 40,41 & 162 & 39,23 & 100,0 \\
\hline S. adleri & 1 & 0,83 & 1 & 0,34 & 2 & 0,48 & 14,2 \\
\hline S. affinis vorax & 1 & 0,83 & 0 & 0,00 & 1 & 0,24 & 14,2 \\
\hline S. clydei & 2 & 1,65 & 16 & 5,48 & 18 & 4,36 & 57,1 \\
\hline Total & 121 & 100,00 & 292 & 100,00 & 413 & 100,00 & \\
\hline
\end{tabular}

Nb. Phl. = Nombre de phlébotomes.

Tableau IV. - Effectif et abondance des captures des pièges lumineux CDC à Palo Youga et à Palo Dial.

testés sans détermination spécifique préalable, pour recherche d'arbovirus. Aucune souche virale n'a été isolée des Phlébotomes de cette zone.

\section{DISCUSSION}

I 'activité des phlébotomes est maximale durant la première moitié de la saison sèche (décembre le Ferlo, région sahélienne dans le nord du Sénégal (Trouillet et al., 1995). Le pic d'abondance de S. buxtoni était plus précoce que ceux de $S$. dubia et de S. schwetzi.

$P$. duboscqi, vecteur de la leishmaniose cutanée humaine (Dedet et al., 1980a) est capturé pour la première fois dans cette région; Ranque (1973), lors de ses missions de juillet-août 1970 et juillet 1971, ne l'avait pas récolté. En revanche il avait été capturé tous les mois de l'année à Keur Moussa situé à environ $27 \mathrm{~km}$ de la zone (Desjeux \& Waroquy, 1981b). Le seul autre Phlebotomus capturé est $P$. rodhaini. Il est également rare. Le rôle éventuel de ces deux espèces de Pblebotomus dans la transmission de Leishmania infantum (agent de la leishmaniose viscérale humaine) signalée au Sénégal n'est toujours pas élucidé. S. dubia, vecteur de Leishmania tarentolae senegalensis (Sauroleishmania) (Desjeux \& Waroquy, 1981a), confirme sa large répartition et sa facilité d'adaptation. $S$. schwetzi, espèce à comportement trophique polyvalent, est très abondante dans les terriers. S. buxtoni, est moins abondant dans le Ferlo qu'à Mont-Rolland, mais dans les deux régions il est plus inféodé aux termitières qu'aux autres biotopes (Trouillet et al., 1995).
Les abondances globales obtenues sont à rapprocher de celles présentées par Basimike et al. (1992) au Kenya ou de Trouillet et al. (1995) dans le Ferlo, où les peuplements sont respectivement composés de 97,3 et $98,7 \%$ d'individus du genre Sergentomyia pour 2,7 et $1,3 \%$ d'individus du genre Phlebotomus. Presque absent dans les captures au piège adhésif, le sousgenre Grassomyia est assez bien représenté dans les captures au piège lumineux CDC. L'abondance des espèces de ce sous-genre est donc liée à la technique de piégeage utilisée comme l'ont déjà montré des études antérieures (Abonnenc, 1972; Desjeux \& Waroquy, 1981; Ba, 1994).

Tous les abris ne sont pas colonisés avec la même intensité. La densité permet de distinguer des gites préférentiels pour certaines espèces. A Mont-Rolland, il existe des différences de densité entre les trous d'arbres et les termitières. Ceci est en accord avec les observations de Dedet et al. $(1978,1980$ b) et de Desjeux et Waroquy (1981b) qui trouvent les termitières de la région de Thiès et de la vallée du fleuve Sénégal assez peu fréquentées par rapport aux trous d'arbres. Par contre, Raymond et Cornet (1976) au Sénégal Oriental, Asimeng (1992) au Nigeria, Basimike et al. (1992), Maranga et al. (1994) au Kenya et Trouillet et al. (1995) au Ferlo ont montré que la densité est plus élevée dans les termitières que dans les terriers et les trous d'arbres. Dans cette étude, la forte densité obtenue dans les trous d'arbres s'explique par le rendement spectaculaire d'un seul gite de ce biotope qui a produit environ $95 \%$ des récoltes des trous d'arbres. Lors de nos captures, même en période d'activité maximale des Phlébotomes, les pièges lumineux n'ont pas permis de récolter plus de cinq cents individus 
pour la recherche d'arbovirus. Cela est peut être lié à des déplacements limités en dehors des gites dans cette région souvent très ventée.

L'absence d'isolement d'arbovirus peut être liée au faible nombre de lots testés (21 lots constitués par 2114 Phlébotomes). Pour la même période d'étude, 60 souches de six virus différents ont été isolées de Phlébotomes des régions de Kédougou et du Ferlo (Thonnon, 1997).

\section{REMERCIEMENTS}

L es auteurs remercient le docteur J.P. Digoutte, le docteur J.P. Moreau, directeur de l'Institut Pasteur de Dakar, et monsieur le président de la communauté rurale de Mont-Rolland pour le soutien constant apporté au cours de cette étude. Ils remercient également messieurs Khalilou Ba, Mamoudou Diallo, Henri Manga et Mathurin Diatta pour leur aide technique.

Travail financé par l'Institut Français de Recherche Scientifique pour le Développement en coopération (ORSTOM), par l'Institut Pasteur de Dakar et par le Ministère Français de la Coopération.

\section{RÉFÉRENCES}

Abonnenc E. Les phlébotomes de la région éthiopienne (Diptera, Psychodidae). Mémoire ORSTOM, 1972, 55, 289 p.

ABOnNEnC E. \& LARIVIère M. Répartition des Phlébotomes de l'Ouest africain (Diptera, Psychodidae). Bulletin de l'Institut fondamental de l'Afrique noire, 1959, sér. A, 21, 204226 .

Asimeng E.J. Natural habitats of phlebotomine sandflies in northern Nigeria. Insect Science and its Application, 1992, 13, 113-119.

BA Y. Dynamique des populations des phlébotomes du Ferlo: implication dans la transmission des arbovirus. Mémoire de D.E.A. de Biologie animale. Université Cheikh Anta Diop de Dakar, 1994, 56, 86 p.

Bameul F. Le DMHF : un excellent milieu de montage en entomologie. L'Entomologiste, 1990, 46, 233-239.

Basimike M., Mutinga M.J. \& Kumar R. Habitat preference and seasonal variations of phlebotomines sandflies (Diptera, Psychodidae) in Marigat area, Baringo District, Kenya. Insect Science and its Application, 1992, 13, 307-314.

Davidson I.H. Sandflies of Africa South of the sahara. Taxonomy and Systematics of the genus Sergentomyia. Johannesburg. The South African Institute for Medical Research. 1990, $75 \mathrm{p}$.

Dedet J.P., Derouin F. \& Cornet M. Infestation spontanée de Pblebotomus duboscqi par des promastigotes de Leishmania au Sénégal. Comptes Rendus des Séances de l'Académie des Sciences de Paris, 1978, sér. D, 286, 301-302.
Dedet J.P., Desjeux P. \& Derouin F. Ecologie d'un foyer de leishmaniose cutanée dans la région de Thiès (Sénégal, Afrique de l'Ouest). 4. Infestation spontanée et biologie de Phlebotomus duboscqi Neveu-Lemaire,1906. Bulletin de la Société de Pathologie exotique, 1980a, 73, 266-276.

Dedet J.P., Winshall R., Hayes R.D. \& Desjeux P. Les Phlébotomes (Diptera, Psychodidae) de la vallée du fleuve Sénégal. Première mention de Sergentomyia (Parvidens) lesleyae Lewis \& Kirk, 1946 en Afrique de l'Ouest. Annales de Parasitologie Humaine et Comparée, 1980b, 55, 125-133.

Desjeux P. \& Waroquy L. Mise en évidence du cycle évolutif de la leishmaniose du Gecko Tarentola anmularis (Geoffroy Saint-Hilaire, 1823) au Sénégal. Rôle vecteur de Sergentomyia dubia (Parrot, Mornet \& Cadenat, 1945). Afrique Médicale, 1981a, 19, 439-442.

Desjeux P. \& Waroquy L. Étude entomologique de 30000 phlébotomes (Diptera, Psychodidae) du Sénégal. Infestation spontanée par Trypanosomatidae. Afrique Médicale, $1981 b, 20,347-352$.

Fontenille D., Traore-lamizana M., Troulllet J., Leclerc A., Mondo M., Ba Y., Digoutte J.P. \& Zeller H.G. First isolations of arboviruses from phlebotomine sand flies in West Africa. American Journal of Tropical Medicine and Hygiene, 1994, 50, 570-574.

Maranga R.O., Irungu L.W. \& Mutinga M.J. Investigations into phlebotomine sandflies in the Nairobi area. Insect Science and its Application, 1994, 15, 145-153.

NiAng A.A. Étude bio-écologique de Phlebotomus duboscqi Neveu-Lemaire, 1906 (Diptera, Psychodidae), vecteur de la leismaniose cutanée humaine au Sénégal. Thèse de Doctorat de Spécialité (3e cycle) de Biologie Animale. Université Cheikh Anta Diop de Dakar,1992, Dakar, Sénégal, $148 \mathrm{p}$.

Niang A.A. \& Trouillet J. Phlébotomes du Sénégal. La faune phlébotomienne du campus universitaire de Dakar (Diptera, Psychodidae). Bulletin de la Société française de Parasitologie, 1993, 11, 151-157.

Niang A.A. \& Trouillet J. Phlébotomes du Sénégal (Diptera, Psychodidae). Faune de la vallée du fleuve Sénégal. Bulletin de l'Institut fondamental de l'Afrique noire Cheikh Anta Diop, Dakar, 1995, 48, 67-78.

PASTRE J. Un nouveau Phlébotome du Sénégal Oriental : Sergentomyia corneti $\mathrm{n}$. sp. (Diptera, Phlebotomidae). Cabiers ORSTOM, série Entomologie médicale et Parasitologie, 1975 , 13, 121-123.

PASTRE J. Quatre espèces nouvelles du sous-genre Sintonius (Diptera, Phlebotomidae, Sergentomyia), avec clé de détermination des espèces éthiopiennes. Cabiers ORSTOM, série Entomologie médicale et Parasitologie, 1982, 20, 231245 .

RANQUE P. Études morphologique et biologique de quelques trypanosomides récoltés au Sénégal. Thèse de Doctorat ès Sciences Naturelles, Université Aix-Marseille II, 1973, CNRS $\mathrm{N}^{\circ}$ A.O. 8223,378 p.

RAYMond H.L. \& Cornet M. Phlébotomes (Diptera, Psychodidae) des termitières du Sénégal Oriental. Annales de Parasitologie Humaine et Comparée, 1976, 51, 259262. 
Thonnon J. Rapport 1996 du Centre Collaborateur OMS de Référence et de Recherche pour les Arbovirus et les virus de fièvres hémorragiques (CRORA). Rapport annuel de l'Institut Pasteur de Dakar, 1997, pp 37-55.

Trouillet J., Ba Y., Traore-Lamizana M., Zeller H.G. \& FonTENILLE D. Phlébotomes (Diptera-Psychodidae) du Sénégal. Peuplement du Ferlo. Isolement d'arbovirus. Parasite, 1995, 2, 289-296.

Trouillet J. \& Faye O. Phlébotomes du Sénégal. Présence de Pblebotomus (Phlebotomus) bergeroti Parrot, 1934 (Diptera, Psychodidae). Annales de Parasitologie humaine et Comparée, 1993, 68, 101-103.

Troulllet J., Vattier-Bernard G. \& Itoua A. Phlébotomes du Sénégal. Description du mâle de Sergentomyia corneti Pastre, 1975 (Diptera, Psychodidae). Bulletin de la société entomologique de France, 1993, 98, 263-266.

Reçu le 10 octobre 1997 Accepté le 12 janvier 1998 